PROCEEDINGS OF THE

AMERICAN MATHEMATICAL SOCIETY

Volume 130, Number 8, Pages 2369-2376

S 0002-9939(02)06339-6

Article electronically published on February 4, 2002

\title{
ABSOLUTELY CONTINUOUS JACOBI OPERATORS
}

\author{
STEEN PEDERSEN \\ (Communicated by David R. Larson)
}

\begin{abstract}
We show (among other results) that a symmetric Jacobi matrix whose diagonal is the zero sequence and whose super-diagonal $h_{n}>0$ satisfies $h_{2 n-1}=h_{2 n}, h_{k} \leq h_{k+1}$ and $0<b \leq \frac{h_{2 k+2}}{k+1} \leq \frac{h_{2 k}}{k}$ has purely absolutely continuous spectrum when considered as a self-adjoint operator on $\ell^{2}(\mathbb{N})$.
\end{abstract}

\section{INTRODUCTION}

The Jacobi operators considered in this paper are operators $H$ acting on the Hilbert space $\ell^{2}(\mathbb{N})$ with the inner product

$$
\langle\phi \mid \psi\rangle=\sum_{n=1}^{\infty} \overline{\phi(n)} \psi(n) .
$$

The operator $H$ is determined by a sequence $h=\left(h_{n}\right), h_{n}>0$ and its action on a vector $\psi \in \ell^{2}(\mathbb{N})$ is determined by

$$
(H \psi)(n)=h_{n-1} \psi(n-1)+h_{n} \psi(n+1) .
$$

If $\left(\xi_{n}=\xi(n)\right)_{n=1}^{\infty}$ is a sequence in some vector space, then set $\xi_{n}=\xi(n)=0$ if $n \leq 0$.

The domain of $H$ is the maximal domain $D(H)=\left\{\psi \in \ell^{2}(\mathbb{N}): H \psi \in \ell^{2}(\mathbb{N})\right\}$. We will impose conditions on $h$ ensuring that $H$ is the closure of its restriction to the minimal domain $\ell_{c}(\mathbb{N})$, the sequences with finite support.

There has recently been considerable interest in the study of spectral properties of Jacobi matrices; see e.g., dMS98, dMS99, Cla96, [DP97, [JM00, JJN99a, [JL99], KL00], [LS99]. Many more references can be found in the recent book Tes00.

As advertised in the abstract, the aim in this note is to prove

Theorem 1.1. If $H$ is determined by a sequence $h_{k}>0$, satisfying $\frac{n}{h_{n}}$ is bounded, $h_{2 n-1}=h_{2 n}$, and

$$
h_{2 n} \leq h_{2 n+2} \leq \frac{n+1}{n} h_{2 n},
$$

then the spectral measure for $H$ is purely absolutely continuous.

Received by the editors September 1, 2000 and, in revised form, March 21, 2001.

2000 Mathematics Subject Classification. Primary 33C45, 39A70; Secondary 47A10, 47 B39.

Key words and phrases. Orthogonal polynomials, weighted shift, absolute continuity, Jacobi matrix. 
The condition $h_{2 n-1}=h_{2 n}>0$ implies that $H$ is essentially self-adjoint on the set of finite sequences (see DP98]), so there is a unique spectral measure associated to $H$.

Theorem [1.1 extends results from DP98 where we proved the result when $h_{2 n-1}=h_{2 n}=n$. Theorem 1.1 is not a consequence of results by Janas and Naboko (e.g. JN99a, JN99b); their bounded variation conditions are not compatible with $h_{2 n-1}=h_{2 n}$ even when $h_{2 n}=n$. The situation where $h_{2 n-1}=h_{2 n}=n+g(n)$ and $\frac{g(n)}{n}$ is bounded is of interest because it is known [HL78, [DP97] that if $\frac{h_{k}}{k} \rightarrow \infty$, then $H$ has pure point spectrum. It is also a boundary case in another sense; see Section 4 below.

This paper began as an attempt to use Mourre's conjugate operator method (e.g., Mou81, CFKS87]) in the context of Jacobi operators determined by a sequence $h_{n}$ with $h_{2 n-1}=h_{2 n}$. Let $a_{n}$ be a sequence of real numbers, and let $A$ be the skew-symmetric Jacobi operator determined by

$$
(A \psi)(n)=-a_{n-1} \psi(n-1)+a_{n} \psi(n+1) .
$$

The case where the double commutator $[[H, A], A]$ is bounded is the simplest case in which one could hope to use Mourre's method. It is easy to see that if the double commutator $[[H, A], A]$ is bounded, then $A$ must be bounded. If $A$ is bounded, then the method used in [DP98] (and in earlier papers by the authors of that paper) is superior to Mourre's method. Positivity of $[H, A]$ is required in a weaker sense by the method from [DP98]. We will therefore use the method from [DP98]. The method from DP98 is an extension due to Dombrowski of a commutator method developed by Putnam Put67. See Section 2.

This paper is motivated by a desire to extend the results from [DP98] to the case where $h_{2 n-1}=h_{2 n}=n+g(n)$ and $g(n)$ is of order less than one in the sense that $\varepsilon_{n}=\frac{g(n)}{n}$ is bounded. Writing $c_{n}=1+\varepsilon_{n}$, we have $h_{2 n}=n c_{n}$, where $c_{n} \neq 0$. At this point the only condition imposed on $c_{n}$ is $c_{n} \neq 0$; further conditions will be imposed below.

\section{The COMmutator Method}

This section will summarize Dombrowski's commutator method as it is currently understood. We will first state the method in an abstract form and then give some typical special cases. In particular, we will show that the method is stronger than what JM00 might lead one to believe. The focus will be on the case where (as in (1.1)) the matrix of the Jacobi operator $H$ has zero diagonal with respect to the canonical basis for $\ell^{2}(\mathbb{N})$.

Theorem 2.1. Let $H$ be a self-adjoint operator on a Hilbert space $\mathcal{H}$ and let $\psi_{0} \in$ $\mathcal{H}$. Let I be a open interval. Suppose there exists a bounded operator $A$ on $\mathcal{H}$ and a constant $b>0$ so that

$$
\left\langle A E(\Delta) \psi_{0} \mid H E(\Delta) \psi_{0}\right\rangle-\left\langle H E(\Delta) \psi_{0} \mid A^{*} E(\Delta) \psi_{0}\right\rangle \geq b\left\|E(\Delta) \psi_{0}\right\|^{4}
$$

for all bounded subintervals $\Delta$ of $I$. Then the restriction of the spectral measure $\mu_{\psi_{0}}(\cdot):=\left\|E(\cdot) \psi_{0}\right\|^{2}$ to the interval $I$ is absolutely continuous with respect to Lebesgue measure on $I$. 
Proof of Theorem [2.1. Let $\mu(\beta):=\left\|E(\beta) \psi_{0}\right\|^{2}$ be the scalar spectral measure associated to $\psi_{0}$. Denote Lebesgue measure by $m$. We will show that

$$
\mu(\beta) \leq C m(\beta)
$$

for all Borel subsets $\beta$ of the interval $I$.

The vector $E(\Delta) \psi_{0}$ is in the domain of $H$ since $\Delta$ is bounded. Let $\lambda$ be the mid-point of $\Delta$. Then

$$
\begin{aligned}
& \left\langle A E(\Delta) \psi_{0} \mid H E(\Delta) \psi_{0}\right\rangle-\left\langle H E(\Delta) \psi_{0} \mid A^{*} E(\Delta) \psi_{0}\right\rangle \\
& =\left\langle A E(\Delta) \psi_{0} \mid(H-\lambda) E(\Delta) \psi_{0}\right\rangle-\left\langle(H-\lambda) E(\Delta) \psi_{0} \mid A^{*} E(\Delta) \psi_{0}\right\rangle \\
& \leq\left\|A E(\Delta) \psi_{0}\right\|\left\|(H-\lambda) E(\Delta) \psi_{0}\right\|+\left\|(H-\lambda) E(\Delta) \psi_{0}\right\|\left\|A^{*} E(\Delta) \psi_{0}\right\| \\
& \leq m(\Delta)\|A\|\left\|E(\Delta) \psi_{0}\right\|^{2}
\end{aligned}
$$

where the last inequality used $\left\|A^{*}\right\|=\|A\|$ and

$$
\left\|(H-\lambda) E(\Delta) \psi_{0}\right\| \leq \frac{1}{2} m(\Delta)\left\|E(\Delta) \psi_{0}\right\| .
$$

Hence $b\left\|E(\Delta) \psi_{0}\right\|^{2} \leq m(\Delta)\|A\|$. Since a Borel set can be approximated by a disjoint union of open intervals, we get (2.2) for all Borel subsets $\beta$ of $I$.

Below, we will write $\langle[H, A] \phi \mid \psi\rangle$ as shorthand for $\langle A \phi \mid H \psi\rangle-\left\langle H \phi \mid A^{*} \psi\right\rangle$ for all $\phi$ and $\psi$ in the domain of $H$.

In this paper we will only use skew-adjoint bounded operators $A$, i.e., only $A$ 's satisfying $A^{*}=-A$ will be used. More precisely, $A$ will be given by (1.3) for suitable $a_{n}$ 's.

When considering Jacobi operators as in (1.1) we will use $\psi_{0}=\delta_{1}$ as the first canonical basis vector for $\ell^{2}(\mathbb{N})$ without further mention. The vector $\delta_{1}$ is a cyclic vector for a Jacobi operator, hence in this case, Theorem 2.1 leads to absolute continuity.

A different modification of Putnam's Theorem is proved in JM00. Their version also allows $H$ to be unbounded; it is closer to Putnam's Theorem in that it requires $\langle[H, A] \psi \mid \psi\rangle>0$ for all $\psi \neq 0$. Note that (2.1) may hold even if $\langle[H, A] \psi \mid \psi\rangle<0$ for some $\psi \neq 0$. This will be the case in the proof of Theorem 1.1 below.

The Mourre method requires that $\langle[H, A] E(I) \psi \mid E(I) \psi\rangle \geq b\|E(I) \psi\|^{2}$ for all $\psi \in \mathcal{H}$. In (2.1) we only need $\psi=E(\Delta) \psi_{0}$ for subintervals $\Delta$ of $I$. On the other hand the Mourre method allows $A$ to be unbounded.

The 4th power in (2.1) is explained by the proof of Corollary 2.2 below.

Dombrowski's choices for $A$ are motivated by considering $H$ to be the real part of a weighted shift $S$ and choosing $A$ to be closely related to the imaginary part of $S$. In this paper it is shown that different choices for $A$ may work better in certain circumstances.

Corollary 2.2. Let $h_{n}$ be a positive sequence and suppose $H$ given by (1.1) is a selfadjoint operator. Suppose there exists a bounded sequence $a_{n}$ of real numbers such that either:

(i) $k_{1,1}>0, k_{n, n} \geq 0$, and $k_{n, n} k_{n+2, n+2} \geq k_{n, n+2}^{2}$ or

(ii) $k_{n, n+2} \geq 0,2 k_{1,1}>k_{1,3}$ and $2 k_{n, n} \geq k_{n, n-2}+k_{n, n+2}$.

Then $H$ has purely absolutely continuous spectrum. Here $k_{n, n}:=h_{n} a_{n}-h_{n-1} a_{n-1}$ and $k_{n+2, n}=k_{n, n+2}:=h_{n+1} a_{n}-h_{n} a_{n+1}$. 
Proof. Let $\Delta$ be any bounded interval and let $A$ be given by (1.3). Let $\delta_{n} \in \ell^{2}(\mathbb{N})$ be the sequence with a 1 in the $n$th position and zeros everywhere else. Hence, $\left(\delta_{n}\right)_{n=1}^{\infty}$ is the canonical basis for $\ell^{2}(\mathbb{N})$.

Let $K:=[H, A]=H A-A H$ and let $x_{n}:=\left\langle E(\Delta) \delta_{1} \mid \delta_{n}\right\rangle$. Then

$$
K \delta_{n}=k_{n, n-2} \delta_{n-2}+2 k_{n, n} \delta_{n}+k_{n, n+2} \delta_{n+2} .
$$

This is the reason for the choice of subscripts in $k_{n, n}$ and $k_{n, n+2}$ in the statement of this corollary.

The reader may verify that

$$
\begin{aligned}
& \left\langle K E(\Delta) \delta_{1} \mid E(\Delta) \delta_{1}\right\rangle=\sum_{m=1}^{\infty}\left\langle K E(\Delta) \delta_{1} \mid \delta_{m}\right\rangle\left\langle\delta_{m} \mid E(\Delta) \delta_{1}\right\rangle \\
& =k_{1,1} x_{1}^{2}+k_{2,2} x_{2}^{2}+\sum_{n=1}^{\infty} k_{n, n} x_{n}^{2}+k_{n+2, n+2} x_{n+2}^{2}+2 k_{n, n+2} x_{n} x_{n+2} .
\end{aligned}
$$

Thus it follows from $(i)$ that

$$
\left\langle K E(\Delta) \delta_{1} \mid E(\Delta) \delta_{1}\right\rangle \geq k_{1,1} x_{1}^{2}=k_{1,1}\left\|E(\Delta) \delta_{1}\right\|^{4} .
$$

Hence $H$ is purely absolutely continuous by Theorem 2.1 .

Suppose (ii). Let $K^{\prime}$ be given by

$$
K^{\prime} \delta_{n}=k_{n, n-2} \delta_{n-2}-2 k_{n, n} \delta_{n}+k_{n, n+2} \delta_{n+2}
$$

and let $U$ be given by $U \delta_{n}=(-1)^{\left\lfloor\frac{n-1}{2}\right\rfloor} \delta_{n}$; then $U K U=-K^{\prime}$. The idea is that $K^{\prime} \leq 0$, by a modification of a well-known criterion (e.g., Ber68, p. 505), and therefore $K \geq 0$. Mimicking the calculation from the proof of Theorem 2.2 in DP95] it follows that

$$
\begin{aligned}
\left\langle K^{\prime} \psi \mid \psi\right\rangle & =\sum_{n=1}^{\infty}\left(k_{n, n-2}-2 k_{n, n}+k_{n, n+2}\right) \psi(n)^{2}-k_{n, n+2}(\psi(n+2)-\psi(n))^{2} \\
& \leq \sum_{n=1}^{\infty}\left(k_{n, n-2}-2 k_{n, n}+k_{n, n+2}\right) \psi(n)^{2} .
\end{aligned}
$$

Setting $\psi=E(\Delta) \delta_{1}$ it follows from (ii) and $U K U=-K^{\prime}$ that $\left\langle K E(\Delta) \delta_{1} \mid E(\Delta) \delta_{1}\right\rangle$ $\geq\left(k_{1,1}-k_{1,3}\right) x_{1}^{2}$. Thus $H$ is purely absolutely continuous by Theorem 2.1

Corollary 2.2 $(i)$ is implicit in much of the work by Dombrowski. Corollary 2.2 ( (ii) has been suspected by Dombrowski and the author for some time; it is a generalization of Theorem 1.2 in [JM00].

\section{Proof of Theorem 1.1}

Theorem 1.1 is an easy consequence of

Theorem 3.1. Let $h_{2 n-1}=h_{2 n}>0$ and let $H$ be determined by (1.1). Suppose there exists a bounded sequence $a_{2 n-1}=a_{2 n}$ such that $g(n):=h_{2 n} a_{2 n}-$ $h_{2 n-2} a_{2 n-2} \geq 1$ and $|f(n)| \leq 1$, where $f(n):=h_{2 n+2} a_{2 n}-h_{2 n} a_{2 n+2}$. Then $H$ is self-adjoint on its maximal domain and the spectral measure for $H$ is purely absolutely continuous. 
Proof. It follows from [DP98] that $H$ is essentially self-adjoint, when considered as an operator with domain $\ell_{c}$, the sequences with finite support. We will also denote the self-adjoint closure by $H$. In fact, the closure of $H$ is the adjoint of $H_{\text {minimal }}$ and is therefore defined on the maximal domain.

Fix $\varepsilon>0$. Let $\Delta$ be an open subinterval of the interval $(\varepsilon, \infty)$.

It is easy to see that $H$ is unitarily equivalent to $-H$. In fact, $-H=U H U$ where $U \delta_{n}=(-1)^{n-1} \delta_{n}$. Hence, $U E(\Delta) U=E(-\Delta)$, where $E$ denotes the projection valued spectral measure associated to $H$. It follows that

$$
0=\left\langle E(\Delta) \delta_{1} \mid E(-\Delta) \delta_{1}\right\rangle=\left\langle E(\Delta) \delta_{1} \mid U E(\Delta) \delta_{1}\right\rangle=\sum_{k=1}^{\infty}(-1)^{k-1}\left|\left\langle E(\Delta) \delta_{1} \mid \delta_{k}\right\rangle\right|^{2}
$$

and therefore

$$
\sum_{n=1}^{\infty}\left|\left\langle E(\Delta) \delta_{1} \mid \delta_{2 n-1}\right\rangle\right|^{2}=\sum_{n=1}^{\infty}\left|\left\langle E(\Delta) \delta_{1} \mid \delta_{2 n}\right\rangle\right|^{2}
$$

The equality (3.1) was observed in [DP98, with the same proof.

The reader may verify that $K=[H, A]=H A-A H$ is given by

$$
\begin{aligned}
K \delta_{2 n-1} & =2 g(n) \delta_{2 n-1} \\
K \delta_{2 n} & =f(n-1) \delta_{2 n-2}+f(n) \delta_{2 n+2} .
\end{aligned}
$$

Let $x_{m}:=\left\langle E(\Delta) \delta_{1} \mid \delta_{m}\right\rangle$. Then

$$
\begin{aligned}
\left\langle K E(\Delta) \delta_{1} \mid E(\Delta) \delta_{1}\right\rangle & =\sum_{m=1}^{\infty}\left\langle K E(\Delta) \delta_{1} \mid \delta_{m}\right\rangle\left\langle\delta_{m} \mid E(\Delta) \delta_{1}\right\rangle \\
& =\sum_{n=1}^{\infty} 2 g(n) x_{2 n-1}^{2}+2 \sum_{n=1}^{\infty} f(n) x_{2 n} x_{2 n+2} \\
& \geq 2 \sum_{n=1}^{\infty} x_{2 n-1}^{2}-2 \sum_{n=1}^{\infty}\left|x_{2 n} x_{2 n+2}\right| \\
& \geq 2 \sum_{n=1}^{\infty} x_{2 n-1}^{2}+x_{2}^{2}-2 \sum_{n=1}^{\infty} x_{2 n}^{2} \\
& =x_{2}^{2}
\end{aligned}
$$

where the last equality used (3.1). Now $H \delta_{1}=h_{1} \delta_{2}$, and therefore $E(\Delta) \delta_{2}=$ $h_{1}^{-1} E(\Delta) H \delta_{1}$. Using $E(\Delta) H \geq \varepsilon E(\Delta)$ and (3.2) it follows that

$$
\left\langle K E(\Delta) \delta_{1} \mid E(\Delta) \delta_{1}\right\rangle \geq \frac{1}{2}\left|\left\langle E(\Delta) \delta_{1} \mid h_{1}^{-1} \varepsilon E(\Delta) \delta_{1}\right\rangle\right|^{2} .
$$

Since $\left\langle E(\Delta) \delta_{1} \mid E(\Delta) \delta_{1}\right\rangle=\left\|E(\Delta) \delta_{1}\right\|^{2}$ it follows that (2.1) holds with $b=\frac{\varepsilon^{2}}{2 h_{1}^{2}}$. Hence $\mu$ is absolutely continuous in $(\varepsilon, \infty)$ by Theorem 2.1. Since $\varepsilon>0$ is arbitrary $\mu$ is absolutely continuous in $(0, \infty)$. Using $-H=U H U$ it follows that $\mu$ is absolutely continuous in $(-\infty, 0) \cup(0, \infty)$.

It remains to show that 0 is not an eigenvalue for $H$. It was observed in DP98 that this is a consequence of the assumption $h_{2 n-1}=h_{2 n}$. In fact, if $\psi \in \ell^{2}(\mathbb{N})$ solves $H \psi=0$, then $\psi(2 n)=0$ and $\psi(2 n-1)=(-1)^{n+1} \psi(1)$; hence $\psi=0$ and therefore 0 is not an eigenvalue for $H$. 
Proof of Theorem 1.1. Suppose $h_{2 n-1}=h_{2 n}=n c_{n}$, for $n>0$, where $c_{n}>0$. Let $H$ be the corresponding self-adjoint Jacobi operator determined by (1.1). Let $A$ be the skew-symmetric Jacobi operator determined by (1.3) where

$$
a_{2 n-1}=a_{2 n}=c_{n}^{-1} \text {. }
$$

Assume $c_{n} \geq b>0$ so that $A$ is bounded. The reader may verify that $K=[H, A]=$ $H A-A H$ is given by

$$
\begin{aligned}
K \delta_{2 n-1} & =2 \delta_{2 n-1} \\
K \delta_{2 n} & =f(n-1) \delta_{2 n-2}+f(n) \delta_{2 n+2},
\end{aligned}
$$

where

$$
f(n):=(n+1) \frac{c_{n+1}}{c_{n}}-n \frac{c_{n}}{c_{n+1}} .
$$

The choice for $a_{n}$ in (3.3) is designed to make $K \delta_{2 n-1}=2 \delta_{2 n-1}$, i.e., $g(n)=1$ for all $n$.

Now $|f(n)| \leq 1$ is equivalent to

$$
-c_{n} c_{n+1}-c_{n+1}^{2} \leq n\left(c_{n+1}^{2}-c_{n}^{2}\right) \leq c_{n} c_{n+1}-c_{n+1}^{2} .
$$

It is easy to see that (3.5) holds if and only if

$$
n c_{n} \leq(n+1) c_{n+1} \text { and } c_{n+1} \leq c_{n} .
$$

This is (1.2) up to a change in notation. An application of Theorem 3.1 completes the proof.

In the following result we allow the entries in $H$ to be negative.

Corollary 3.2. Suppose $h_{2 n-1}=h_{2 n}, h_{1} \neq 0$, and $h_{2 n+1}=h_{1} \cdot(n+1) \cdot \prod_{k=1}^{n} \eta_{n}$. If $\frac{n}{n+1} \leq\left|\eta_{n}\right| \leq 1$ and $\prod_{n=1}^{\infty}\left|\eta_{n}\right|>0$, then $H$ is absolutely continuous.

Proof. Up to unitary equivalence we may assume that all entries of $H$ are nonnegative, hence the corollary is a direct consequence of the proof of Theorem 1.1 see in particular (3.6).

\section{Concluding COMments}

4.1. No doubles. We will now state the analogue of Theorem 1.1 in the case where the assumption $h_{2 n-1}=h_{2 n}$ is removed.

Theorem 4.1. Let $h_{n}>0$ satisfy that $\frac{n}{h_{n}}$ is bounded and

$$
h_{n} \leq h_{n+1} \leq \frac{n+1}{n} h_{n} .
$$

Then $H$ given by (1.1) is self-adjoint on its maximal domain and its spectral measure is purely absolutely continuous.

Proof. It is an immediate consequence of (4.1) that $\sum h_{n}^{-1}=\infty$, hence $H$ is selfadjoint by Carleman's criterion. Let $c_{n}=\frac{h_{n}}{n}$. Let $f(n)$ be given by (3.4). It follows as in the proof of Theorem 1.1 that $|f(n)| \leq 1$. Let $A$ be given by (1.3) with $a_{n}=c_{n}^{-1}$. The sequence $a_{n}$ is positive and non-increasing, hence bounded. The reader may verify that $K=H A-A H$ is given by

$$
K \delta_{n}=f(n-2) \delta_{n-2}+2 \delta_{n}+f(n) \delta_{n+2} .
$$

Since $|f(n)| \leq 1$ it follows from Corollary 2.2 that the spectral measure for $H$ is absolutely continuous with respect to Lebesgue measure. 
Note that $h_{2 n-1}=h_{2 n}$ implies $c_{2 n-1}=\frac{2 n}{2 n-1}$ and $c_{2 n}=1$. Hence, Theorem 1.1 is not contained in this result.

Theorem 4.1] was proved in [JM00] with (4.1) replaced by the stronger assumption

$$
\sqrt{\frac{n+1}{n}} h_{n} \leq h_{n+1} \leq \frac{n+1}{n} h_{n}
$$

The paper [JM00 compares various approaches to the study of spectral properties of Jacobi operators. One of the conclusions reached there is that their version of Theorem 4.1 "seem to be quite strong tools for spectral studies. This theorem works also for some cases which cannot be studied by subordination methods."

4.2. The square. In DP95 the authors suggested studying spectral properties of Jacobi matrices whose row sums all are equal to zero. A direct consequence of Theorem 1.1 and [DP97] is:

Theorem 4.2. Suppose $h_{n}>0$ satisfies $h_{n} \leq h_{n+1}$ and $0<b \leq \frac{h_{n+1}}{(n+1)^{2}} \leq \frac{h_{n}}{n^{2}}$. Let $H$ be given by

$$
H \delta_{n}=h_{n-1} \delta_{n}-\left(h_{n-1}+h_{n}\right) \delta_{n}+h_{n} \delta_{n+1} .
$$

Then $H$ is self-adjoint on its maximal domain and its spectral measure is purely absolutely continuous.

It follows from the work of Janas and Naboko [JN99a, JN99b] that if $H$ is given by $H \delta_{n}=h_{n-1} \delta_{n}-g_{n} \delta_{n}+h_{n} \delta_{n+1}$ and $\frac{g_{n}}{h_{n}}=c$, then (in many cases) $H$ has purely discrete spectrum if $c>2$ and purely absolutely continuous spectrum if $c<2$. Theorem 4.2 is a boundary case because there $c=2$.

\section{REFERENCES}

[Ber68] Ju.M. Berezanskii, Expansion in Eigenfunctions of Selfadjoint Operators, Translations of Mathematical Monographs, vol. 17, Amer. Math. Soc., Providence, RI, 1968. MR 36:5768

[CFKS87] H.L. Cycon, R.G. Froese, W. Kirsch, and B. Simon, Schrödinger operators and applications to quantum mechanichs and global geometry, Texts and Monographs in Physics, Springer-Verlag, Berlin-New York, 1987. MR 88g:35003

[Cla96] S.L. Clark, A spectral analysis for self-adjoint operators generated by a class of secondorder difference equations, J. Math. Anal. Appl. 197 (1996), 267-285. MR 96m:47061

[dMS98] A. Boutet de Monvel and J. Sahbani, On the spectral properties of the spin-boson Hamiltonians, Lett. Math. Phys. 44 (1998), 23-33. MR 2000m:81047

[dMS99] A. Boutet de Monvel and J. Sahbani, On the spectral properties of discrete Schrödinger operators: the multi-dimensional case, Rev. Math. Phys. 11 (1999), 1061-1078. MR 2000j: 47064

[DP95] J. Dombrowski and S. Pedersen, Orthogonal polynomials, spectral measures and absolute continuity, J. Comput. Appl. Math. 65 (1995), 115-124. MR 97b:47027

[DP97] J. Dombrowski and S. Pedersen, Spectral measures and Jacobi matrices related to Laguerre type systems of orthogonal polynomials, Constr. Approx. 13 (1997), 421-433. MR 98e:47041

[DP98] J. Dombrowski and S. Pedersen, Absolute continuity for Jacobi matrices with constant row sums, J. Math. Anal. Appl., to appear.

[HL78] D.B. Hinton and R.T. Lewis, Spectral analysis of second-order difference equations, J. Math. Anal. Appl. 63 (1978), 421-438. MR 58:29512

[JL99] S. Jitomirskaya and Y. Last, Power-law subordinacy and singular spectra I. Half-line operators, Acta Math. 183 (1999), 171-189. MR 2001a:47033

[JM00] J. Janas and M. Moszyński, The alternative approaches to the absolute continuity of Jacobi matrices with monotonic weights, Integral Equations Operator Theory, to appear. 
[JN99a] J. Janas and S. Naboko, Jacobi matrices with power-like weights - grouping in blocks approach, J. Funct. Anal. 166 (1999), 218-243. MR 2000k:47032

[JN99b] J. Janas and S. Naboko, Multitreshold spectral phase transition examples in a class of unbounded Jacobi matrices, Recent advances in operator theory (Groningen, 1998), 267-285. Oper. Theory Adv. Appl., 124, Birkhäuser, Basel, 2001.

[KL00] A. Kiselev and Y. Last, Solutions, spectrum and dynamics for Schrödinger operators on infinite domains, Duke Math. J. 102 (2000), 125-150. CMP 2000:09

[LS99] Y. Last and B. Simon, Eigenfunctions, transfer matrices, and absolutely continuous spectrum of one-dimensional Schrödinger operators, Invent. Math. 135 (1999), 329367. MR 2000f: 47060

[Mou81] E. Mourre, Absence of singular continuous spectrum for certain selfadjoint operators, Commun. Math. Phys. 78 (1981), 391-408. MR 82c:47030

[Put67] C.R. Putnam, Commutation Properties of Hilbert Space Operators and Related Topics, Ergebnisse der Math., vol. 36, Springer-Verlag, Berlin, 1967. MR 36:707

[Tes00] G. Teschl, Jacobi Operators and Completely Integrable Nonlinear Latices, Mathematical Surveys and Monograhps, vol. 72, Amer. Math. Soc., Providence, RI, 2000. MR 2001b:39019

Department of Mathematics, Wright State University, Dayton, Ohio 45435

E-mail address: steen@math.wright.edu 